\title{
Stable Strong Fenchel and Lagrange duality for evenly convex optimization problems*
}

\author{
M.D. FAJARDO ${ }^{1}$, J. VIDAL \\ Department of Statistics and Operations Research \\ University of Alicante, 03080 Alicante, Spain
}

\begin{abstract}
By means of a conjugation scheme based on generalized convex conjugation theory instead of Fenchel conjugation, we build an alternative dual problem, using the perturbational approach, for a general optimization one defined on a separated locally convex topological space. Conditions guaranteeing strong duality for disturbed primal problems by continuous linear functionals and their respective dual problems, which is named stable strong duality, are stablished. In these conditions, the evenly convexity of the perturbation function will play a fundamental role. Stable strong duality will also be studied in particular for Fenchel and Lagrange primal-dual problems, obtaining a characterization for Fenchel case.
\end{abstract}

Keywords: Evenly convex function, generalized convex conjugation, Lagrange and Fenchel dual problems.

Mathematics Subject Classification (2010): 52A20, 26B25, 90C25.

${ }^{1}$ Corresponding author. E-mail adresses: md.fajardo@ua.es and jose.vidal@ua.es.

* This research was partially supported by MICINN of Spain, Grant MTM2011-29064-C03-02 and by Conselleria de la Eduacion de la Generalitat Valenciana, Spain, Pre-doc Program Vali+d, DOCV 6791/07.06.2012, Grant ACIF-2013-156.

\section{Introduction}

There exists a fundamental approach to duality for studying the general optimization problem

$$
\begin{array}{lll}
(G P) & \text { Inf } & F(x) \\
& \text { s.t. } & x \in X,
\end{array}
$$

where $F: X \rightarrow \overline{\mathbb{R}}:=\mathbb{R} \cup\{ \pm \infty\}$, by a perturbation function $\Phi: X \times \Theta \rightarrow \overline{\mathbb{R}}$, such that $\Phi(x, 0)=F(x)$, for all $x \in X$. In this case, a dual problem associated to $(G P)$, verifying weak duality, which means that the optimal value of the primal problem is greater or equal to the optimal value of the dual one, is given by

$$
\begin{aligned}
& (G D) \text { Sup }-\Phi^{*}\left(0, z^{*}\right) \\
& \text { s.t. } z^{*} \in \Theta^{*} \text {, }
\end{aligned}
$$


where $X$ and $\Theta$ are separated locally convex spaces and $\Phi^{*}: X^{*} \times \Theta^{*} \rightarrow \overline{\mathbb{R}}$ is the Fenchel conjugate function of $\Phi$. One of the most interesting problems in optimization theory is to find conditions under which there exists strong duality, i.e.

$$
\inf _{x \in X} \Phi(x, 0)=\max _{z^{*} \in \Theta^{*}}-\Phi^{*}\left(0, z^{*}\right)
$$

which means that there is not duality gap and the dual problem is solvable. These conditions are called regularity conditions. However, from the point of view of applicability, it is necessary to find conditions guaranteeing strong duality even when the objective function of the primal problem $(G P)$ is disturbed with linear continuous functionals, situation which is named stable strong duality.

Sufficient interior point-type conditions for stable strong duality can be found in [18], while it is characterized by a much more general closedness-type condition in [2]. Sufficient and necessary conditions for stable strong Fenchel and Fenchel-Lagrange duality are derived in [11] and [1], respectively. Similar characterizations for stable strong Lagrange dualtity are also given in [8] and [1]. In most of them, the lower semicontinuity and convexity of the function $\Phi$ (or, when the perturbational approach is not used, of the involved functions in the primaln problem) are necessary. This framework is what we have called, in our work, the classical context.

Evenly convex functions (e-convex function, en brief), can be viewed as a generalization of convex lower semicontinuous functions. This kind of functions arose ([17]), in a natural way, from the concept of evenly convex set (e-convex set, in brief), defined as an intersection of an arbitrary family of open half-spaces, and due to Fenchel ([4]). In [6], it can be found some well-known results for lower semicontinuous convex functions which were extended to that more general framework. Since that paper, our challenge has been to find regularity conditions for strong duality with the assumption of e-convexity of the perturbation function (see also [5], [7]). Fenchel conjugation is not a suitable scheme for e-convex functions, in the sense that biconjugating by Fenchel an e-convex function produces a lower semicontinuous convex function which is not necessarily the original one, but this is not the case of c-conjugation (see [15]). This conjugation scheme has been used in all our work in order to obtain a general dual problem for $(G P)$ instead of $(G D)$, as well as dual problems for particular cases of $(G P)$, like Fenchel and Lagrange primal-dual problems. 
The aim of this paper is to continue developing duality c-conjugation theory by finding conditions for stable strong duality. The organization is as follows. In Section 2 we summarize the basic properties for e-convex functions, as well as all the necessary tools and results, in order to make the paper self-contained. In particular, the conjugation scheme for e-convex functions will be reminded, as well as its most important properties. Moreover, we will recall how the dual problem $\left(G D_{c}\right)$ for $(G P)$ is built by means of c-conjugation. In Section 3 we will show that two regularity conditions for strong duality for $(G P)-\left(G D_{c}\right)$ are also sufficient conditions for stable strong duality. Section 4 is devoted to the particular case of stable strong Fanchel duality, which is going to be characterized, while Section 5 presents sufficient conditions in the case of stable strong Lagrange duality.

\section{Preliminaries}

Consider $X$ a separated locally convex space and $X^{*}$ its topological dual space endowed with the weak* topology induced by $X$. For a set $D \subset X$ (resp. $D \subset X^{*}$ ), the closure of $D$ (resp. weak* closure of $D$ ) is denoted by $\mathrm{cl} D$, and the notation $\delta_{D}$ will stand for the indicator function of $D$. By $\left\langle x, x^{*}\right\rangle$ we denote $x^{*}(x)$ for all $\left(x, x^{*}\right) \in X \times X^{*}$. According to [3], a set $C \subset X$ is e-convex if for every $x_{0} \notin C$, there exists $x^{*} \in X^{*}$ such that $\left\langle x-x_{0}, x^{*}\right\rangle<0$, for all $x \in C$. An application of Hahn-Banach theorem is that every open or closed convex set is e-convex. The e-convex hull, eco $K$, of a set $K \subset X$ is the smallest e-convex set that contains $K$. This operator is well defined because $X$ is e-convex and the class of e-convex sets is closed under intersection. Moreover, if $K$ is convex, then $K \subset$ eco $K \subset \operatorname{cl} K$. Another property which appears in [9] for finite dimensional spaces and can be also shown easily in the infinite dimensional case is that the cartesian product of a finite number of e-convex sets is also an e-convex set in the product space.

For a function $f: X \rightarrow \overline{\mathbb{R}}$, we denote $\operatorname{dom} f=\{x \in X \mid f(x)<+\infty\}$ the effective domain of $f$ and by epi $f=\{(x, r) \in X \times \mathbb{R} \mid f(x) \leq r\}$ its epigraph. We call $f$ proper if $\operatorname{dom} f \neq \emptyset$ and $f(x)>-\infty$, for all $x \in X$.

The lower semicontinuous (lsc, in short) hull of $f, \operatorname{cl} f: X \rightarrow \overline{\mathbb{R}}$, is defined such that epi $(\operatorname{cl} f)=\operatorname{cl}($ epi $f)$, and $f$ is said to be lsc at $x \in X$ if $f(x)=(\operatorname{cl} f)(x)$. On the other hand, according to [17], we will say that $f$ is e-convex if its epigraph is an e-convex set in $X \times \mathbb{R}$. Clearly, any lsc convex function is e-convex, but the converse statement is not true (see an example in [17]). The e-convex hull of $f$, eco $f: X \rightarrow \overline{\mathbb{R}}$, is defined as the largest e-convex 
minorant of $f$, that is,

$$
\text { eco } f:=\sup \{g \mid g \text { is e-convex and } g \leq f\} \text {. }
$$

According to [17, Proposition 3.1], if $f: X \rightarrow \overline{\mathbb{R}}$ is an e-convex function and $\alpha>0$, then $\alpha f$ is an e-convex function, and by [17, Proposition 3.3], if $f, g: X \rightarrow \overline{\mathbb{R}}$ are two proper e-convex functions with $\operatorname{dom} f \cap \operatorname{dom} g \neq \emptyset$, then $f+g$ is also an e-convex function (see [6, page 381, footnote 1]).

Definition 2.1 A function $a: X \rightarrow \overline{\mathbb{R}}$ is said to be e-affine if there exist $x^{*}, y^{*} \in X^{*}$ and $\alpha, \beta \in \mathbb{R}$ such that

$$
a(x)= \begin{cases}\left\langle x, x^{*}\right\rangle-\beta & \text { if }\left\langle x, y^{*}\right\rangle<\alpha \\ +\infty & \text { otherwise. }\end{cases}
$$

For any $f: X \rightarrow \overline{\mathbb{R}}, \mathcal{E}_{f}$ denotes the set of all e-affine functions minorizing $f$, that is,

$$
\mathcal{E}_{f}:=\{a: X \rightarrow \overline{\mathbb{R}} \mid \text { a is } \text { e-affine and } a \leq f\}
$$

From [15] we have the following characterization for a proper e-convex function.

Theorem 2.1 Let $f: X \rightarrow \overline{\mathbb{R}}$, $f$ not identically $+\infty$ or $-\infty$. Then $f$ is a proper e-convex function if and only if

$$
f=\sup \left\{a \mid a \in \mathcal{E}_{f}\right\}
$$

Based on the generalized convex conjugation theory introduced by Moreau [16], a suitable conjugation scheme is provided in [15] for e-convex functions. Consider the set $W:=X^{*} \times$ $X^{*} \times \mathbb{R}$ with the coupling functions $c: X \times W \rightarrow \overline{\mathbb{R}}$ and $c^{\prime}: W \times X \rightarrow \overline{\mathbb{R}}$ given by

$$
c\left(x,\left(x^{*}, y^{*}, \alpha\right)\right)=c^{\prime}\left(\left(x^{*}, y^{*}, \alpha\right), x\right):=\left\{\begin{array}{cl}
\left\langle x, x^{*}\right\rangle & \text { if }\left\langle x, y^{*}\right\rangle<\alpha, \\
+\infty & \text { otherwise. }
\end{array}\right.
$$

For a function $f: X \rightarrow \overline{\mathbb{R}}$, its $c$-conjugate $f^{c}: W \rightarrow \overline{\mathbb{R}}$ is defined by

$$
f^{c}\left(\left(x^{*}, y^{*}, \alpha\right)\right):=\sup _{x \in X}\left\{c\left(x,\left(x^{*}, y^{*}, \alpha\right)\right)-f(x)\right\} .
$$

Similarly, the $c^{\prime}$-conjugate of a function $g: W \rightarrow \overline{\mathbb{R}}$ is $g^{c^{\prime}}: X \rightarrow \overline{\mathbb{R}}$ defined by

$$
g^{c^{\prime}}(x):=\sup _{\left(x^{*}, y^{*}, \alpha\right) \in W}\left\{c^{\prime}\left(\left(x^{*}, y^{*}, \alpha\right), x\right)-g\left(x^{*}, y^{*}, \alpha\right)\right\}
$$

with the conventions $(+\infty)+(-\infty)=(-\infty)+(+\infty)=(+\infty)-(+\infty)=(-\infty)-(-\infty)=$ $-\infty$. 
Functions of the form $x \in X \rightarrow c\left(x,\left(x^{*}, y^{*}, \alpha\right)\right)-\beta \in \overline{\mathbb{R}}$, with $\left(x^{*}, y^{*}, \alpha\right) \in W$ and $\beta \in \mathbb{R}$ are called c-elementary; in the same way, $c^{\prime}$-elementary functions are those of the form $\left(x^{*}, y^{*}, \alpha\right) \in W \rightarrow c\left(x,\left(x^{*}, y^{*}, \alpha\right)\right)-\beta \in \overline{\mathbb{R}}$, with $x \in X$ and $\beta \in \mathbb{R}$.

In [15] it is shown that the family of the proper e-convex functions from $X$ to $\overline{\mathbb{R}}$ along with the function identically equal to $-\infty$ is actually the family of pointwise suprema of sets of celementary functions. Using an analogous terminology, a function $g: W \rightarrow \overline{\mathbb{R}}$ is said $e^{\prime}$-convex if it is the pointwise supremum of sets of $\mathrm{c}^{\prime}$-elementary functions. Also, the $e^{\prime}$-convex hull of any function $k: W \rightarrow \overline{\mathbb{R}}$ is the largest $\mathrm{e}^{\prime}$-convex minorant of $k$, and it is denoted by $\mathrm{e}^{\prime}$ co $k$. The following proposition is given in [14].

Proposition 2.1 Let $f: X \rightarrow \overline{\mathbb{R}}$ and $g: W \rightarrow \overline{\mathbb{R}}$. Then

(i) $f^{c}$ is $e^{\prime}$-convex; $g^{c^{\prime}}$ is e-convex.

(ii) If $f$ has a proper e-convex minorant, eco $f=f^{c c^{\prime}} ; \mathrm{e}^{\prime} \operatorname{co} g=g^{c^{\prime} c}$.

(iii) If $f$ does not take on the value $-\infty$, then $f$ is e-convex if and only if $f=f^{c c^{\prime}} ; g$ is $e^{\prime}$-convex if and only if $g=g^{c^{\prime} c}$.

(iv) $f^{c c^{\prime}} \leq f ; g^{c^{\prime}} \leq g$.

The following definitions from [6] will be needed in the sequel.

Definition 2.2 A set $D \subset W \times \mathbb{R}$ is $\mathrm{e}^{\prime}$-convex if there exists an $e^{\prime}$-convex function $k: W \rightarrow \overline{\mathbb{R}}$ such that $D=$ epi $k$. The $\mathrm{e}^{\prime}$-convex hull of an arbitrary set $D \subset W \times \mathbb{R}$ is defined as the smallest $e^{\prime}$-convex set containing $D$, and it will be denoted by $\mathrm{e}^{\prime} \mathrm{co} D$.

Definition 2.3 Consider two functions $f, g: X \rightarrow \overline{\mathbb{R}}$. A function $a: X \rightarrow \overline{\mathbb{R}}$ belongs to the set $\widetilde{\mathcal{E}}_{f, g}$ if there exist $a_{1} \in \mathcal{E}_{f}, a_{2} \in \mathcal{E}_{g}$ such that, if

$$
a_{1}(\cdot)=\left\{\begin{array}{ll}
\left\langle\cdot, x_{1}^{*}\right\rangle-\beta_{1} & \text { if }\left\langle\cdot, y_{1}^{*}\right\rangle<\alpha_{1}, \\
+\infty & \text { otherwise, }
\end{array} \text { and } a_{2}(\cdot)= \begin{cases}\left\langle\cdot, x_{2}^{*}\right\rangle-\beta_{2} & \text { if }\left\langle\cdot, y_{2}^{*}\right\rangle<\alpha_{2}, \\
+\infty & \text { otherwise, }\end{cases}\right.
$$

then

$$
a(\cdot)= \begin{cases}\left\langle\cdot, x_{1}^{*}+x_{2}^{*}\right\rangle-\left(\beta_{1}+\beta_{2}\right) & \text { if }\left\langle\cdot, y_{1}^{*}+y_{2}^{*}\right\rangle<\alpha_{1}+\alpha_{2} \\ +\infty & \text { otherwise. }\end{cases}
$$

Finally, associated to a general optimization problem, expressed in terms of an appropiate perturbation function

$$
\begin{array}{lll}
(G P) & \operatorname{Inf} & \Phi(x, 0) \\
& \text { s.t. } & x \in X,
\end{array}
$$


we will use the following dual problem, obtained in [6], by means of c-conjugation

$$
\begin{aligned}
(G D) \operatorname{Sup} & -\Phi^{c}\left(\left(0, u^{*}\right),\left(0, v^{*}\right), \alpha\right) \\
& u^{*}, v^{*} \in X^{*}
\end{aligned}
$$

and weak duality is guaranteed.

\section{Stable strong duality}

We introduce the concept of stable strong duality considering the extension of the general problem

$$
\begin{aligned}
\left(G P_{x^{*}}\right) \quad \text { Inf } & \Phi(x, 0)+\left\langle x, x^{*}\right\rangle \\
\text { s.t. } & x \in X
\end{aligned}
$$

for an arbitrary $x^{*} \in X^{*}$. This means that we disturb the general problem with a linear functional, and a pertubation function for $\left(G P_{x^{*}}\right)$ will be $\Phi_{x^{*}}: X \times \Theta \rightarrow \overline{\mathbb{R}}$ defined as

$$
\Phi_{x^{*}}(x, u):=\Phi(x, u)+\left\langle x, x^{*}\right\rangle .
$$

C-conjugating $\Phi_{x^{*}}$ makes it possible to associate to $\left(G P_{x^{*}}\right)$ a dual problem verifying weak duality. Letting $Z=X \times \Theta$, the appropriate coupling function for building the c-conjugate of $\Phi_{x^{*}}$ will be $c_{1}: Z \times Z^{*} \times Z^{*} \times \mathbb{R} \rightarrow \overline{\mathbb{R}}$ such that

$$
c_{1}\left((x, u),\left(\left(y^{*}, u^{*}\right),\left(z^{*}, v^{*}\right), \alpha\right)\right)= \begin{cases}\left\langle x, y^{*}\right\rangle+\left\langle u, u^{*}\right\rangle & \text { if }\left\langle x, z^{*}\right\rangle+\left\langle u, v^{*}\right\rangle<\alpha \\ +\infty & \text { otherwise. }\end{cases}
$$

We have $\Phi_{x^{*}}^{c}: Z^{*} \times Z^{*} \times \mathbb{R} \rightarrow \overline{\mathbb{R}}$ and

$$
\begin{aligned}
\Phi_{x^{*}}^{c}\left(\left(y^{*}, u^{*}\right),\left(z^{*}, v^{*}\right), \alpha\right)= & \sup _{(x, u) \in Z}\left\{c_{1}\left((x, u),\left(\left(y^{*}, u^{*}\right),\left(z^{*}, v^{*}\right), \alpha\right)\right)-\Phi_{x^{*}}(x, u)\right\} \\
= & \sup _{(x, u) \in Z}\left\{c_{1}\left((x, u),\left(\left(y^{*}, u^{*}\right),\left(z^{*}, v^{*}\right), \alpha\right)\right)-\Phi(x, u)-\left\langle x, x^{*}\right\rangle\right\} \\
= & \sup _{(x, u) \in Z}\left\{c_{1}\left((x, u),\left(\left(y^{*}-x^{*}, u^{*}\right),\left(z^{*}, v^{*}\right), \alpha\right)\right)-\Phi(x, u)\right\} \\
= & \Phi^{c}\left(\left(y^{*}-x^{*}, u^{*}\right),\left(z^{*}, v^{*}\right), \alpha\right) .
\end{aligned}
$$

Then, a dual problem associated with $\left(G P_{x^{*}}\right)$ is, according to (2),

$$
\begin{aligned}
\left(G D_{x^{*}}\right) \text { Sup } \quad-\Phi^{c}\left(\left(-x^{*}, u^{*}\right),\left(0, v^{*}\right), \alpha\right) \\
\quad u^{*}, v^{*} \in \Theta^{*} \\
\text { s.t. } \quad \alpha>0 .
\end{aligned}
$$


Definition 3.1 We say that it holds stable strong duality for $(G P)$ and $(G D)$ if, for all $x^{*} \in X^{*},\left(G P_{x^{*}}\right)$ and $\left(G D_{x^{*}}\right)$ verify strong duality, i.e.,

$$
\operatorname{Inf}_{x \in X} \Phi(x, 0)+\left\langle x, x^{*}\right\rangle=\operatorname{Max}_{\substack{u^{*}, v^{*} \in \Theta^{*}, \alpha>0 .}}-\Phi^{c}\left(\left(-x^{*}, u^{*}\right),\left(0, v^{*}\right), \alpha\right)
$$

We recall the regularity conditions for closing duality gap between $(G P)$ and $(G D)$ being also the last problem solvable which appear in [5], for $\Phi$ being a proper e-convex function. To this aim, it is necessary to introduce the following concepts and notation: letting $Y$ be any arbitrary topological space, $\operatorname{Pr}_{Y}$ is the projection operator onto $Y$, and for any nonempty convex set $A$ contained in $Y$, the algebraic relative interior of $A$ is the set of all the points $a \in A$ verifying cone $(A-a)$ is a linear subspace of $Y$. It is denoted by ${ }^{i} A$, and ${ }^{i c} A$ is the subset of ${ }^{i} A$ whose points verify that cone $(A-a)$ is moreover closed. The mentioned regularity conditions have the following description:

(C1) $X$ and $\Theta$ are Fréchet spaces, and $0 \in{ }^{i c}\left(\operatorname{Pr}_{\Theta} \operatorname{dom} \Phi\right)$.

(C2) $\operatorname{Pr}_{W \times \mathbb{R}}\left(\operatorname{epi} \Phi^{c}\right)$ is $\mathrm{e}^{\prime}$-convex.

Clearly, since $\Phi_{x^{*}}=\Phi+\left\langle\cdot, x^{*}\right\rangle$, we have that $\Phi_{x^{*}}$ is proper and e-convex if and only if $\Phi$ is proper and e-convex. Then, it follows that

$\left(\mathrm{C1}_{x^{*}}\right) X$ and $\Theta$ are Fréchet spaces, and $0 \in{ }^{i c}\left(\operatorname{Pr}_{\Theta} \operatorname{dom} \Phi_{x^{*}}\right)$.

$\left(\mathrm{C} 2_{x^{*}}\right) \operatorname{Pr}_{W \times \mathbb{R}}\left(\operatorname{epi} \Phi_{x^{*}}^{c}\right)$ is $\mathrm{e}^{\prime}$-convex,

for all $x^{*} \in X^{*}$, are, each of them, sufficient conditions for strong duality between $\left(G P_{x^{*}}\right)$ and $\left(G D_{x^{*}}\right)$. Condition $(\mathrm{C} 1)$ is sufficient for the fulfilment of $\left(\mathrm{C}_{x^{*}}\right)$, for all $x^{*} \in X^{*}$ because $\operatorname{dom} \Phi_{x^{*}}=\operatorname{dom} \Phi$, hence $(\mathrm{C} 1)$ implies stable strong duality for $(G P)$ and $(G D)$. The following lemma is necessary for showing that condition $(\mathrm{C} 2)$ is also a sufficient condition for stable strong duality.

Lemma 3.1 Let $C \subset W \times \mathbb{R}$ be a non-empty $e^{\prime}$-convex set. Then, for any $x^{*} \in X^{*}$ and $\delta \in \mathbb{R}$, $C+\left\{\left(x^{*}, 0,0, \delta\right)\right\}$ is $e^{\prime}$-convex.

Proof. Let $x^{*} \in X^{*}, \delta \in \mathbb{R}$ and $C^{\prime}:=C+\left\{\left(x^{*}, 0,0, \delta\right)\right\}$. Name $H: W \rightarrow \overline{\mathbb{R}}$ the pointwise supremum function of a certain set of $\mathrm{c}^{\prime}$-elementary functions, i.e.,

$$
H=\sup _{(x, \beta) \in A}\{c(x, \cdot)-\beta\},
$$

being $A$ a non-empty subset of $X \times \mathbb{R}$, such that

$$
C=\text { epi } H=\bigcap_{(x, \beta) \in A} \operatorname{epi}\{c(x, \cdot)-\beta\} .
$$


Then

$C^{\prime}=\left[\bigcap_{(x, \beta) \in A} \operatorname{epi}\{c(x, \cdot)-\beta\}\right]+\left\{\left(x^{*}, 0,0, \delta\right)\right\}=\bigcap_{(x, \beta) \in A}\left[\operatorname{epi}\{c(x, \cdot)-\beta\}+\left\{\left(x^{*}, 0,0, \delta\right)\right\}\right]$.

Now, we have, for any $(x, \beta) \in A$,

$$
\begin{aligned}
& \operatorname{epi}\{c(x, \cdot)-\beta\}+\left\{\left(x^{*}, 0,0, \delta\right)\right\} \\
= & \left\{\left(y^{*}, z^{*}, \alpha, \gamma\right) \in W \times \mathbb{R} \mid\left\langle x, y^{*}-x^{*}\right\rangle-\beta \leq \gamma-\delta,\left\langle x, z^{*}\right\rangle<\alpha\right\} \\
= & \left\{\left(y^{*}, z^{*}, \alpha, \gamma\right) \in W \times \mathbb{R} \mid\left\langle x, y^{*}\right\rangle-\left(\beta+\left\langle x, x^{*}\right\rangle+\delta\right) \leq \gamma,\left\langle x, z^{*}\right\rangle<\alpha\right\} .
\end{aligned}
$$

We define the following set in $X \times \mathbb{R}$

$$
\widehat{A}=\left\{(x, \widehat{\beta}) \mid \widehat{\beta}=\beta+\left\langle x, x^{*}\right\rangle+\delta \text { and }(x, \beta) \in A\right\} .
$$

We obtain that, for any $(x, \beta) \in A$,

$$
\text { epi }\{c(x, \cdot)-\beta\}+\left\{\left(x^{*}, 0,0, \delta\right)\right\}=\operatorname{epi}\{c(x, \cdot)-\widehat{\beta}\}
$$

and $C^{\prime}=\bigcap_{(x, \widehat{\beta}) \in \widehat{A}} \operatorname{epi}\{c(x, \cdot)-\widehat{\beta}\}$ is $\mathrm{e}^{\prime}$-convex.

In general, we cannot asure that the sum of an $\mathrm{e}^{\prime}$-convex set with any point will be again an $\mathrm{e}^{\prime}$-convex set.

Example 3.1 Let $X=\mathbb{R}$. We claim that any non-empty e'-convex set $K$ in $\mathbb{R}^{4}$ must verify that the boundary of its proyection onto $\mathbb{R}^{2}$ corresponding to the second and third coordinates contains the origin, $\mathrm{O}_{2}$.

Indeed, if $K=\bigcap_{(a, b) \in A \times B}$ epi $\{c(a, \cdot)-b\}$, where $A \times B \subset \mathbb{R}^{2}$, it is clear that $\operatorname{Pr}_{\mathbb{R}^{2}}(K)$ is the solution set of the system

$$
\{a x-y<0, a \in A\} .
$$

The announced property is due to [9, Prop.1.1].

Now, take the $\mathrm{e}^{\prime}$-convex set $C=\left\{(y, z, \alpha, \gamma) \in \mathbb{R}^{4} \mid y \leq \gamma, z<\alpha\right\}=\operatorname{epi} c(1, \cdot)$, and let $C^{\prime}=C+\{(1,1,0,0)\}$. Then

$$
C^{\prime}=\left\{(x, y, \alpha, \beta) \in \mathbb{R}^{4} \mid x+\beta \leq 1, y+\alpha<1\right\},
$$

and $\operatorname{Pr}_{\mathbb{R}^{2}}\left(C^{\prime}\right)=\left\{(y, \alpha) \in \mathbb{R}^{2}|y+\alpha<1|\right\}$, so $0_{2}$ belongs to the interior of this projection, meaning that $C^{\prime}$ is not $\mathrm{e}^{\prime}$-convex.

Proposition 3.1 (C2) is a sufficient condition for stable strong duality. 
Proof. Let us check that (C2) implies the fulfiment of $\left(\mathrm{C} 2 x_{x^{*}}\right)$, for all $x^{*} \in X^{*}$. The fact is that

$$
\operatorname{Pr}_{W \times \mathbb{R}}\left(\operatorname{epi} \Phi_{x^{*}}^{c}\right)=\operatorname{Pr}_{W \times \mathbb{R}}\left(\operatorname{epi} \Phi^{c}\right)+\left\{\left(x^{*}, 0,0,0\right)\right\}
$$

and, in virtue of Lemma 3.1, the proof is completed.

Let $x^{*} \in X^{*}$. A point $\left(\left(y^{*}, u^{*}\right),\left(z^{*}, v^{*}\right), \alpha, \beta\right)$ belongs to epi $\Phi_{x^{*}}^{c}$ if and only if the corresponding point $\left(\left(y^{*}-x^{*}, u^{*}\right),\left(z^{*}, v^{*}\right), \alpha, \beta\right)$ belongs to epi $\Phi^{c}$. We have

$$
\left(\left(y^{*}, u^{*}\right),\left(z^{*}, v^{*}\right), \alpha, \beta\right)=\left(\left(y^{*}-x^{*}, u^{*}\right),\left(z^{*}, v^{*}\right), \alpha, \beta\right)+\left(\left(x^{*}, 0\right),(0,0), 0,0\right),
$$

for each point $\left(\left(y^{*}, u^{*}\right),\left(z^{*}, v^{*}\right), \alpha, \beta\right) \in \operatorname{epi} \Phi_{x^{*}}^{c}$, then

$$
\text { epi } \Phi_{x^{*}}^{c} \subset \text { epi } \Phi^{c}+\left\{\left(\left(x^{*}, 0\right),(0,0), 0,0\right)\right\} \text {. }
$$

The reversed inclusion is analogous. Consequently, (3) holds.

There is no relationship between conditions (C1) and (C2), and unfortunately none of them characterizes stable strong duality, as we will see in the two following sections, where Fenchel and Lagrange duality problems are studied.

\section{Fenchel Duality Problem}

Let us consider the following optimization problem

$$
\begin{array}{lll}
(P) & \text { Inf } & f(x) \\
& \text { s.t. } & x \in A,
\end{array}
$$

where $A \subset X$ is a non-empty e-convex set and $f: X \rightarrow \overline{\mathbb{R}}$ is a proper e-convex function, with $\operatorname{dom} f \cap A \neq \emptyset$. The problem $(P)$ is a particular case of $(G P)$ with $F=f+\delta_{A}$, where $\delta_{A}$ is the indicator function of $A$.

Taking the perturbation function $\Phi: X \times X \rightarrow \overline{\mathbb{R}}$ given by

$$
\Phi(x, u):= \begin{cases}f(x+u) & \text { if } x \in A \\ +\infty & \text { otherwise }\end{cases}
$$

the dual problem for $(P)$ was obtained in [6] as

$$
\begin{aligned}
&\left(D_{F}\right) \operatorname{Sup} \quad\left\{-f^{c}\left(u^{*}, v^{*}, \alpha_{1}\right)-\delta_{A}^{c}\left(-u^{*},-v^{*}, \alpha_{2}\right)\right\} \\
& \text { s.t. } \quad u^{*}, v^{*} \in X^{*}, \\
& \alpha_{1}+\alpha_{2}>0,
\end{aligned}
$$

together with the following closedness-type regularity condition: 
$\left(\mathrm{C}_{F}\right) f+\delta_{A}=\sup \left\{a \mid a \in \widetilde{\mathcal{E}}_{f, \delta_{A}}\right\}$ and epi $f^{c}+\operatorname{epi} \delta_{A}^{c}$ is $\mathrm{e}^{\prime}$-convex.

Now, let us consider the extended primal and dual problems, for all $x^{*} \in X^{*}$,

$$
\begin{aligned}
& \left(P_{x^{*}}\right) \quad \operatorname{Inf} f(x)+\left\langle x, x^{*}\right\rangle \\
& \text { s.t. } x \in A \text {, } \\
& \left(D_{F, x^{*}}\right) \quad \operatorname{Sup} \quad\left\{-\left(f+\left\langle\cdot, x^{*}\right\rangle\right)^{c}\left(u^{*}, v^{*}, \alpha_{1}\right)-\delta_{A}^{c}\left(-u^{*},-v^{*}, \alpha_{2}\right)\right\} \\
& u^{*}, v^{*} \in X^{*} \text {, } \\
& \text { s.t. } \quad \alpha_{1}+\alpha_{2}>0 \text {. }
\end{aligned}
$$

One has

$$
\begin{aligned}
\left(f+\left\langle\cdot, x^{*}\right\rangle\right)^{c}\left(u^{*}, v^{*}, \alpha_{1}\right) & =\sup _{x \in x}\left\{c\left(x,\left(u^{*}, v^{*}, \alpha_{1}\right)\right)-f(x)-\left\langle x, x^{*}\right\rangle\right\} \\
& =\sup _{x \in x}\left\{c\left(x,\left(u^{*}-x^{*}, v^{*}, \alpha_{1}\right)\right)-f(x)\right\} \\
& =f^{c}\left(u^{*}-x^{*}, v^{*}, \alpha_{1}\right)
\end{aligned}
$$

and

$$
\begin{aligned}
\left(D_{F, x^{*}}\right) \operatorname{Sup} \quad\left\{-f^{c}\left(u^{*}-x^{*}, v^{*}, \alpha_{1}\right)-\delta_{A}^{c}\left(-u^{*},-v^{*}, \alpha_{2}\right)\right\} & \\
\text { s.t. } \quad & u^{*}, v^{*} \in X^{*} \\
& \alpha_{1}+\alpha_{2}>0 .
\end{aligned}
$$

It holds stable strong duality for $(P)-\left(D_{F}\right)$ if, for all $x^{*} \in X^{*}$, the following condition were true:

$$
\left(\mathrm{C}_{F, x^{*}}\right) f+\left\langle\cdot, x^{*}\right\rangle+\delta_{A}=\sup \left\{a \mid a \in \widetilde{\mathcal{E}}_{f+\left\langle\cdot, x^{*}\right\rangle, \delta_{A}}\right\} \text { and epi }\left(f+\left\langle\cdot, x^{*}\right\rangle\right)^{c}+\text { epi } \delta_{A}^{c} \text { is } \mathrm{e}^{\prime}-
$$
convex.

\section{Proposition 4.1 Condition $\left(C_{F}\right)$ guarantees stable strong Fenchel duality.}

Proof. Let us show that, for all $x^{*} \in X^{*},\left(\mathrm{C}_{F, x^{*}}\right)$ holds, whenever $\left(\mathrm{C}_{F}\right)$ holds. Take any $x^{*} \in X^{*}$.

In first place, let us assume that $f+\delta_{A}=\sup \left\{a \mid a \in \widetilde{\mathcal{E}}_{f, \delta_{A}}\right\}$. We will check the equality $f+\left\langle\cdot, x^{*}\right\rangle+\delta_{A}=\sup \left\{a \mid a \in \widetilde{\mathcal{E}}_{f+\left\langle\cdot, x^{*}\right\rangle, \delta_{A}}\right\}$.

Let us observe that an e-affine function $a \in \mathcal{E}_{f+\left\langle\cdot x^{*}\right\rangle}$ if and only if $a \leq f+\left\langle\cdot, x^{*}\right\rangle$, which is equivalent to $a-\left\langle\cdot, x^{*}\right\rangle$ be e-ffine and $a-\left\langle\cdot, x^{*}\right\rangle \leq f$, meaning that $a-\left\langle\cdot, x^{*}\right\rangle \in \mathcal{E}_{f}$. It follows immediately that $\widetilde{\mathcal{E}}_{f+\left\langle\cdot, x^{*}\right\rangle, \delta_{A}}=\widetilde{\mathcal{E}}_{f, \delta_{A}}+\left\langle\cdot, x^{*}\right\rangle$ and $\sup \left\{a \mid a \in \widetilde{\mathcal{E}}_{f+\left\langle\cdot, x^{*}\right\rangle, \delta_{A}}\right\}=$ $\sup \left\{a \mid a \in \widetilde{\mathcal{E}}_{f, \delta_{A}}\right\}+\left\langle\cdot, x^{*}\right\rangle=f+\delta_{A}+\left\langle\cdot, x^{*}\right\rangle$. 
In second place, we claim that

$$
\text { epi }\left(f+\left\langle\cdot, x^{*}\right\rangle\right)^{c}=\text { epi } f^{c}+\left(x^{*}, 0,0,0\right) .
$$

Of course, $\left(u^{*}, v^{*}, \alpha, \beta\right) \in$ epi $\left(f+\left\langle\cdot, x^{*}\right\rangle\right)^{c}$ if and only if, for all $x \in X$,

$$
c\left(x,\left(u^{*}, v^{*}, \alpha\right)\right)-f(x)-\left\langle x, x^{*}\right\rangle \leq \beta,
$$

which means that, for all $x \in X$,

$$
c\left(x,\left(u^{*}-x^{*}, v^{*}, \alpha\right)\right)-f(x) \leq \beta,
$$

or, equivalently, $\left(u^{*}-x^{*}, v^{*}, \alpha, \beta\right) \in \operatorname{epi~} f^{c}$.

Hence, from (5), we obtain

$$
\text { epi }\left(f+\left\langle\cdot, x^{*}\right\rangle\right)^{c}+\operatorname{epi} \delta_{A}^{c}=\operatorname{epi} f^{c}+\operatorname{epi} \delta_{A}^{c}+\left(x^{*}, 0,0,0\right),
$$

fact that, according to Lemma 3.1 allows us to say that, if epi $f^{c}+$ epi $\delta_{A}^{c}$ is $\mathrm{e}^{\prime}$-convex, then epi $\left(f+\left\langle\cdot, x^{*}\right\rangle\right)^{c}+\operatorname{epi} \delta_{A}^{c}$ is $\mathrm{e}^{\prime}$-convex.

Our aim is to find a characterization of stable strong duality for $(P)-\left(D_{F}\right)$. Although $\left(\mathrm{C}_{F}\right)$ is a sufficient condition, it is not necessary. In fact, if $\left(\mathrm{C}_{F}\right)$ were equivalent to stable strong duality, in particular, $(\mathrm{C} 2)$ would imply $\left(\mathrm{C}_{F}\right)$, and Example 6.6 in [5] shows that this is not true. We have to look in another direction.

We recall that, for two given functions $g, h: X \rightarrow \overline{\mathbb{R}}$, the infimal convolution of $g$ and $h$, $g \square h: X \rightarrow \overline{\mathbb{R}}$, is defined as

$$
(g \square h)(x):=\inf _{x_{1}+x_{2}=x}\left\{g\left(x_{1}\right)+h\left(x_{2}\right)\right\} .
$$

Moreover, it is said that the infimal convolution is exact a point $a \in X$ if there exists $a_{1}, a_{2} \in$ $X$, with $a_{1}+a_{2}=a$, such that $(g \square h)(a)=g\left(a_{1}\right)+h\left(a_{2}\right)$.

Our characterization is motivated by [11], where in the classical setting, strong Fenchel duality is equivalent to the inequality

$$
\left(f+\delta_{A}\right)^{*}(0) \geq\left(f^{*} \square \delta_{A}^{*}\right)(0),
$$

together with the exactness of the infimal convolution at the point 0 , being $f$ and $\delta_{A}$ proper and convex functions.

Definition 4.1 We say that condition (C3) holds for the problem $(P)$ if there exists $\alpha>0$ such that

$$
\left(f+\delta_{A}\right)^{c}(0,0, \alpha) \geq\left(f^{c} \square \delta_{A}^{c}\right)(0,0, \alpha)
$$


and the infimal convolution is exact at $(0,0, \alpha)$.

In [6] it was shown that, if $v(P) \in \mathbb{R}$ and $f+\delta_{A}=\sup \left\{a \mid a \in \widetilde{\mathcal{E}}_{f, \delta_{A}}\right\}$, condition (C3) is sufficient for strong Fenchel duality. In this work, we will see that also is a characterization of strong Fenchel duality, which will allow us to obtain a characterization of stable strong Fenchel duality. First, we need a lemma.

Lemma 4.1 Condition (C3) holds for $(P)$ with a certain $\alpha>0$ if and only if

$$
\text { epi }\left(f+\delta_{A}\right)^{c} \cap\{(0,0, \alpha) \times \mathbb{R}\} \subseteq\left(\text { epi } f^{c}+\text { epi } \delta_{A}^{c}\right) \cap\{(0,0, \alpha) \times \mathbb{R}\} .
$$

Proof. First, suppose that (C3) holds. Clearly, if epi $\left(f+\delta_{A}\right)^{c} \cap\{(0,0, \alpha) \times \mathbb{R}\}=\emptyset$, there is nothing to prove. Hence, let $(0,0, \alpha, \beta) \in \operatorname{epi}\left(f+\delta_{A}\right)^{c}$ so $\left(f+\delta_{A}\right)^{c}(0,0, \alpha) \leq \beta$. Since we are assuming that $\left(f^{c} \square \delta_{A}^{c}\right)$ is exact at $(0,0, \alpha)$, there exist $u^{*}, v^{*} \in X^{*}, \alpha_{1}, \alpha_{2} \in \mathbb{R}$ with $\alpha_{1}+\alpha_{2}=\alpha$ such that

$$
f^{c}\left(u^{*}, v^{*}, \alpha_{1}\right)+\delta_{A}^{c}\left(-u^{*},-v^{*}, \alpha_{2}\right) \leq \beta,
$$

meaning that $\left(u^{*}, v^{*}, \alpha_{1}, \beta-\delta_{A}^{c}\left(-u^{*},-v^{*}, \alpha_{2}\right)\right) \in \operatorname{epi} f^{c}$.

On the other hand, $\left(-u^{*},-v^{*}, \alpha_{2}, \delta_{A}^{c}\left(-u^{*},-v^{*}, \alpha_{2}\right)\right) \in \operatorname{epi} \delta_{A}^{c}$, because $\delta_{A}^{c}\left(-u^{*},-v^{*}, \alpha_{2}\right)$ is finite, and we can write

$$
(0,0, \alpha, \beta)=\left(u^{*}, v^{*}, \alpha_{1}, \beta-\delta_{A}^{c}\left(-u^{*},-v^{*}, \alpha_{2}\right)\right)+\left(-u^{*},-v^{*}, \alpha_{2}, \delta_{A}^{c}\left(-u^{*},-v^{*}, \alpha_{2}\right)\right),
$$

and $(0,0, \alpha, \beta) \in \operatorname{epi} f^{c}+\operatorname{epi} \delta_{A}^{c}$.

Now, let us suppose that (6) holds for some $\alpha>0$. In the case $v(P)=-\infty$, since $\left(f+\delta_{A}\right)^{c}(0,0, \alpha)=-v(P)$, clearly (C3) fulfils. Then let $v(P)=\delta \in \mathbb{R}$, and hence $\left(f+\delta_{A}\right)^{c}(0,0, \alpha)=-\delta$. We obtain $(0,0, \alpha,-\delta) \in \operatorname{epi}\left(f+\delta_{A}\right)^{c} \cap\{(0,0, \alpha) \times \mathbb{R}\}$. Since (6) is true, there exist $u^{*}, v^{*} \in X^{*}, \alpha_{1}, \alpha_{2}, \delta_{1}, \delta_{2} \in \mathbb{R}$ such that $\alpha_{1}+\alpha_{2}=\alpha, \delta_{1}+\delta_{2}=-\delta$ with $\left(u^{*}, v^{*}, \alpha_{1}, \delta_{1}\right) \in \operatorname{epi} f^{c}$ and $\left(-u^{*},-v^{*}, \alpha_{2}, \delta_{2}\right) \in \operatorname{epi} \delta_{A}^{c}$. Then

$$
\left(f^{c} \square \delta_{A}^{c}\right)(0,0, \alpha) \leq f^{c}\left(u^{*}, v^{*}, \alpha_{1}\right)+\delta_{A}^{c}\left(-u^{*},-v^{*}, \alpha_{2}\right) \leq-\delta,
$$

and we obtain

$$
\left(f^{c} \square \delta_{A}^{c}\right)(0,0, \alpha) \leq\left(f+\delta_{A}\right)^{c}(0,0, \alpha) .
$$

Ir order to see that the infimal convolution is exact at $(0,0, \alpha)$, since $v(D)=-\left(f^{c} \square \delta_{A}^{c}\right)(0,0, \alpha)$, from weak duality and (7), we have

$$
-v(D)=\left(f^{c} \square \delta_{A}^{c}\right)(0,0, \alpha) \leq-v(P) \leq-v(D)
$$


then

$$
\left(f^{c} \square \delta_{A}^{c}\right)(0,0, \alpha)=-v(P)=f^{c}\left(u^{*}, v^{*}, \alpha_{1}\right)+\delta_{A}^{c}\left(-u^{*},-v^{*}, \alpha_{2}\right) .
$$

Proposition 4.2 If $v(P) \in \mathbb{R}$ and $f+\delta_{A}=\sup \left\{a \mid a \in \widetilde{\mathcal{E}}_{f, \delta_{A}}\right\},(C 3)$ is necessary for strong Fenchel duality.

Proof. Let us suppose that $v(P)=v(D)=\delta$ and

$$
f^{c}\left(u^{*}, v^{*}, \alpha_{1}\right)+\delta_{A}^{c}\left(-u^{*},-v^{*}, \alpha_{2}\right)=-\delta,
$$

for certain $u^{*}, v^{*} \in X^{*}, \alpha_{1}, \alpha_{2} \in \mathbb{R}, \alpha_{1}+\alpha_{2}>0$. Name $\alpha:=\alpha_{1}+\alpha_{2}$. We will show (C3) holds for this $\alpha$, through the equivalent condition in Lemma 4.1.

Since $-\delta=\left(f+\delta_{A}\right)^{c}(0,0, \alpha)$, taking any point $(0,0, \alpha, \beta) \in$ epi $\left(f+\delta_{A}\right)^{c}$, we have that $-\delta \leq \beta$, and from $(8)$

$$
f^{c}\left(u^{*}, v^{*}, \alpha_{1}\right)+\delta_{A}^{c}\left(-u^{*},-v^{*}, \alpha_{2}\right) \leq \beta .
$$

Then $\left(u^{*}, v^{*}, \alpha_{1}, \beta-\delta_{A}^{c}\left(-u^{*},-v^{*}, \alpha_{2}\right)\right) \in$ epi $f^{c}$ and $\left(-u^{*},-v^{*}, \alpha_{2}, \delta_{A}^{c}\left(-u^{*},-v^{*}, \alpha_{2}\right)\right) \in$ epi $\delta_{A}^{c}$, obtaining that $(0,0, \alpha, \beta) \in$ epi $f^{c}+\operatorname{epi} \delta_{A}^{c}$.

From the above proposition we have the following characterization of stable strong Fenchel duality, when $f+\delta_{A}=\sup \left\{a \mid a \in \widetilde{\mathcal{E}}_{f, \delta_{A}}\right\}$ :

(C4) For all $x^{*} \in X^{*}, v\left(P_{x^{*}}\right) \in \mathbb{R}$ and there exists $\alpha_{x^{*}}>0$ such that

$$
\left(f+\left\langle\cdot, x^{*}\right\rangle+\delta_{A}\right)^{c}\left(0,0, \alpha_{x^{*}}\right) \geq\left(\left(f+\left\langle\cdot, x^{*}\right\rangle\right)^{c} \square \delta_{A}^{c}\right)\left(0,0, \alpha_{x^{*}}\right),
$$

and the infimal convolution is exact at $\left(0,0, \alpha_{x^{*}}\right)$.

Here we can see, from another point of view, why $\left(\mathrm{C}_{F}\right)$ does not characterize stable strong duality., i.e., (C4) and $\left(\mathrm{C}_{F}\right)$ are not equivalent. More precisely, $(\mathrm{C} 4)$ does not imply that epi $f^{c}+$ epi $\delta_{A}^{c}$ would be $\mathrm{e}^{\prime}$-convex, under the hypothesis of $f+\delta_{A}=\sup \left\{a \mid a \in \widetilde{\mathcal{E}}_{f, \delta_{A}}\right\}$. According to Theorem 11 in [6], the fact of epi $f^{c}+\operatorname{epi} \delta_{A}^{c}$ being $\mathrm{e}^{\prime}$-convex is equivalent to

$$
\left(f+\delta_{A}\right)^{c} \geq\left(f^{c} \square \delta_{A}^{c}\right)
$$

and the infimal convolution being exact at any point. This is the same than

$$
\text { epi }\left(f+\delta_{A}\right)^{c} \subseteq\left(\text { epi } f^{c}+\operatorname{epi} \delta_{A}^{c}\right) \text {. }
$$


From Lemma 4.1, what we can assure from condition (C4) is that, for all $x^{*} \in X^{*}$, there exists $\alpha_{x^{*}}>0$ such that

$$
\operatorname{epi}\left(f+\left\langle\cdot, x^{*}\right\rangle+\delta_{A}\right)^{c} \cap\left\{\left(0,0, \alpha_{x^{*}}\right) \times \mathbb{R}\right\} \subseteq\left(\operatorname{epi}\left(f+\left\langle\cdot, x^{*}\right\rangle\right)^{c}+\operatorname{epi} \delta_{A}^{c}\right) \cap\{(0,0, \alpha) \times \mathbb{R}\}
$$

Since, for any $x^{*} \in X^{*}$ and any function $h: X \rightarrow \overline{\mathbb{R}}$ it holds

$$
\text { epi }\left(h+\left\langle\cdot, x^{*}\right\rangle\right)^{c}=\operatorname{epi} h^{c}+\left\{\left(x^{*}, 0,0,0\right)\right\},
$$

inclusion (9) will be equivalent to the existence, of $\alpha_{x^{*}}>0$, for all $x^{*} \in X^{*}$, such that

$$
\begin{aligned}
& \left(\operatorname{epi}\left(f+\delta_{A}\right)^{c}+\left\{\left(x^{*}, 0,0,0\right)\right\}\right) \cap\left\{\left(0,0, \alpha_{x^{*}}\right) \times \mathbb{R}\right\} \\
\subseteq & \left(\operatorname{epi} f^{c}+\operatorname{epi} \delta_{A}^{c}+\left\{\left(x^{*}, 0,0,0\right)\right\}\right) \cap\{(0,0, \alpha) \times \mathbb{R}\},
\end{aligned}
$$

meaning that, for all $x^{*} \in X^{*}$, there exists $\alpha_{x^{*}}>0$ such that

$$
\begin{aligned}
& \operatorname{epi}\left(f+\delta_{A}\right)^{c} \cap\left\{\left(x^{*}, 0, \alpha_{x^{*}}\right) \times \mathbb{R}\right\} \\
\subseteq & \left(\text { epi } f^{c}+\text { epi } \delta_{A}^{c}\right) \cap\left\{\left(x^{*}, 0, \alpha_{x^{*}}\right) \times \mathbb{R}\right\},
\end{aligned}
$$

fact that, clearly, does not imply epi $\left(f+\delta_{A}\right)^{c} \subseteq\left(\right.$ epi $\left.f^{c}+\operatorname{epi} \delta_{A}^{c}\right)$. We only can guarantee the inclusion of points written as $\left(y^{*}, 0, \alpha, \beta\right)$, with $\alpha>0$ depending on $y^{*}$.

\section{Lagrange Duality Problem}

Let us consider now the following problem

$$
\begin{array}{lll}
(P) & \text { Inf } & f(x) \\
& \text { s.t. } & g_{t}(x) \leq 0, t \in T,
\end{array}
$$

where $f, g_{t}: X \rightarrow \overline{\mathbb{R}}$ are proper e-convex functions, and $T$ is an arbitrary index set. Let us suppose that the feasible set $A=\left\{x \in X \mid g_{t}(x) \leq 0, t \in T\right\}$ is non-empty. Again, the problem $(P)$ is a particular case of $(G P)$ with $F=f+\delta_{A}$. We consider the following perturbation function $\Phi: X \times \mathbb{R}^{T} \rightarrow \overline{\mathbb{R}}$

$$
\Phi(x, b)= \begin{cases}f(x) & \text { if } g_{t}(x) \leq b_{t} \\ +\infty & \text { otherwise }\end{cases}
$$

where the perturbation variable $b=\left(b_{t}\right)_{t \in T} \in \mathbb{R}^{T}$.

Denoting by $\sigma=\left\{g_{t}(x) \leq 0, t \in T\right\}$, we can reformulate $\sigma$ as $\left\{g(x) \in-\mathbb{R}_{+}^{T}\right\}$, where $g: X \rightarrow \overline{\mathbb{R}}^{T}$ is defined as

$$
g(x)(t):=g_{t}(x), \forall t \in T
$$


for all $x \in X$ and the perturbation function $\Phi$ is rewritten

$$
\Phi(x, b)= \begin{cases}f(x) & \text { if } g(x)-b \in-\mathbb{R}_{+}^{T}, \\ +\infty & \text { otherwise }\end{cases}
$$

In this point we recall the so-called space of generalized finite sequences, $\mathbb{R}^{(T)}$, whose elements, $\lambda=\left(\lambda_{t}\right)_{t \in T}$, verify that only finitely many $\lambda_{t}$ are different from zero, i.e., their support set, which is denoted by $\operatorname{supp} \lambda$, is finite.

Taking $\mathbb{R}^{(T)}$ as the dual space of $\mathbb{R}^{T}$, with the following dual product

$$
\lambda b:=\sum_{t \in T} \lambda_{t} b_{t}
$$

for all $\lambda \in \mathbb{R}^{(T)}, b \in \mathbb{R}^{T}$, and letting $Z=X \times \mathbb{R}^{T}$, the appropriate coupling function for building the c-conjugate of $\Phi$ will be $c_{1}: Z \times Z^{*} \times Z^{*} \times \mathbb{R} \rightarrow \overline{\mathbb{R}}$ such that

$$
c_{1}\left((x, b),\left(\left(x^{*}, \lambda\right),\left(y^{*}, \beta\right), \alpha\right)\right)= \begin{cases}\left\langle x, x^{*}\right\rangle+\lambda b & \text { if }\left\langle x, y^{*}\right\rangle+\beta b<\alpha, \\ +\infty & \text { otherwise. }\end{cases}
$$

We have $\Phi^{c}: Z^{*} \times Z^{*} \times \mathbb{R} \rightarrow \overline{\mathbb{R}}$ and

$$
\Phi^{c}\left(\left(x^{*}, \lambda\right),\left(y^{*}, \beta\right), \alpha\right)=\sup _{(x, b) \in Z}\left\{c_{1}\left((x, b),\left(\left(x^{*}, \lambda\right),\left(y^{*}, \beta\right), \alpha\right)\right)-\Phi(x, b)\right\},
$$

and dual problem obtained in [7]

$$
\begin{aligned}
& \left(D_{L}\right) \quad \operatorname{Sup}\left\{\inf _{x \in X}\{f(x)+\lambda g(x)\}\right\} \\
& \text { s.t. } \lambda \in \mathbb{R}_{+}^{(T)} .
\end{aligned}
$$

with the following regularity condition

$\left(\mathrm{C}_{L}\right) \bigcup_{\lambda \in \mathbb{R}_{+}^{(T)}} \operatorname{epi}(\lambda g)^{c}$ is an $\mathrm{e}^{\prime}$-convex set, $f+\delta_{A}=\sup \left\{a \mid a \in \widetilde{\mathcal{E}}_{f, \delta_{A}}\right\}$ and epi $f^{c}+\operatorname{epi} \delta_{A}^{c}$ is $\mathrm{e}^{\prime}$-convex.

Now, let us consider the extended primal and dual problems, for all $x^{*} \in X^{*}$,

$$
\begin{gathered}
\left(P_{x^{*}}\right) \quad \text { Inf } f(x)+\left\langle x, x^{*}\right\rangle \\
\text { s.t. } \quad g_{t}(x) \leq 0, t \in T, \\
\left(D_{L, x^{*}}\right) \quad \operatorname{Sup}\left\{\inf _{x \in X}\left\{f(x)+\left\langle x, x^{*}\right\rangle+\lambda g(x)\right\}\right\} \\
\text { s.t. } \lambda \in \mathbb{R}_{+}^{(T)} .
\end{gathered}
$$

Following the same steps than in the proof of Proposition 4.1, we can conclude that $\left(\mathrm{C}_{L}\right)$ is sufficient also for stable strong duality for $(P)-\left(D_{L}\right)$. 
Again, looking for a possible characterization of stable strong duality...From Example 5.1 in [7], where it is shown that condition $(\mathrm{C} 2)$ does not imply $\left(\mathrm{C}_{L}\right)$, we derive $\left(\mathrm{C}_{L}\right)$ is not necessary for such duality.

In the classical context, with $f$ and $g_{t}$ for all $t \in T$ proper convex and lsc functions, [1] showed that stable strong Lagrange duality is equivalent to the closedness of the set

$$
\bigcup_{\lambda \in \mathbb{R}_{+}^{(T)}} \operatorname{epi}(f+\lambda g)^{*} .
$$

What can we say in our context? Can we extened the result if we talk about $\mathrm{e}^{\prime}$-convexity of the set $\bigcup_{\lambda \in \mathbb{R}_{+}^{(T)}} \operatorname{epi}(f+\lambda g)^{c}$ instead of closedness of $\bigcup_{\lambda \in \mathbb{R}_{+}^{(T)}} \operatorname{epi}(f+\lambda g)^{*}$ ?

\section{Proposition 5.1 It holds}

$$
\bigcup_{\lambda \in \mathbb{R}_{+}^{(T)}} \operatorname{epi}(f+\lambda g)^{c} \subset \operatorname{Pr}{ }_{W \times \mathbb{R}}\left(\operatorname{epi} \Phi^{c}\right) \subset \operatorname{epi}\left(f+\delta_{A}\right)^{c} .
$$

Proof. Let $\left(x^{*}, y^{*}, \alpha, \beta\right) \in \bigcup_{\lambda \in \mathbb{R}_{+}^{(T)}} \operatorname{epi}(f+\lambda g)^{c}$. Then there exists $\lambda \in \mathbb{R}_{+}^{(T)}$ such that, for all $x \in \operatorname{dom} f \cap\left[\cap_{t \in \operatorname{supp} \lambda} \operatorname{dom} g_{t}\right]$,

$$
c\left(x,\left(x^{*}, y^{*}, \alpha\right)\right)-f(x)-\lambda g(x) \leq \beta .
$$

then, if $(x, b) \in X \times \mathbb{R}^{T}$ verifies $x \in \operatorname{dom} f$ and $g(x)-b \in-\mathbb{R}_{+}^{T}$, it is clear that $x \in$ $\cap_{t \in \operatorname{supp} \lambda} \operatorname{dom} g_{t}$, and, moreover, since $\lambda g(x) \leq \lambda b$, from (11), we have

$$
\left\langle x, x^{*}\right\rangle-f(x)-\lambda b \leq \beta
$$

together with $\left\langle x, y^{*}\right\rangle+0 \cdot b<\alpha$, which yields to

$$
c_{1}\left((x, b),\left(x^{*},-\lambda\right),\left(y^{*}, 0\right), \alpha\right)-f(x) \leq \beta .
$$

Due to the fact that this is true for all $(x, b) \in X \times \mathbb{R}^{T}$ such that $x \in \operatorname{dom} f$ and $g(x)-b \in-\mathbb{R}_{+}^{T}$, we conclude that

$$
\Phi^{c}\left(\left(x^{*},-\lambda\right),\left(y^{*}, 0\right), \alpha\right) \leq \beta,
$$

and $\left(x^{*}, y^{*}, \alpha, \beta\right) \in \operatorname{Pr}_{W \times \mathbb{R}}\left(\operatorname{epi} \Phi^{c}\right)$.

The inclusion $\operatorname{Pr}_{W \times \mathbb{R}}\left(\operatorname{epi} \Phi^{c}\right) \subset$ epi $\left(f+\delta_{A}\right)^{c}$ comes from [5, Lemma 5.3], taking into account that $\Phi(\cdot, 0)=f+\delta_{A}$.

Taking $\mathrm{e}^{\prime}$-convex hulls in (10), inequalities are transformed into equalities:

Proposition 5.2 epi $\left(f+\delta_{A}\right)^{c}=\mathrm{e}^{\prime}$ co $\bigcup_{\lambda \in \mathbb{R}_{+}^{(T)}} \operatorname{epi}(f+\lambda g)^{c}$. 
Proof. For this proof, we will apply Remark 2.2 in [6], where the $\mathrm{e}^{\prime}$-convex hull of any set $K \subset W \times \mathbb{R}$ was characterized as the epigraph of the function $h^{c^{\prime} c}$ where $h: W \rightarrow \mathbb{R}$ is defined as

$$
h\left(x^{*}, y^{*}, \alpha\right)=\inf \left\{a \in \mathbb{R} \mid\left(x^{*}, y^{*}, \alpha, \beta\right) \in K\right\} .
$$

Taking $K=\bigcup_{\lambda \in \mathbb{R}_{+}^{(T)}} \operatorname{epi}(f+\lambda g)^{c}$, we have that $\left(x^{*}, y^{*}, \alpha, \beta\right) \in K$ if and only if there exists $\lambda \in \mathbb{R}_{+}^{(T)}$ such that $(f+\lambda g)^{c}\left(x^{*}, y^{*}, \alpha\right) \leq \beta$, hence

$$
h\left(x^{*}, y^{*}, \alpha\right)=\inf \left\{(f+\lambda g)^{c}\left(x^{*}, y^{*}, \alpha\right) \mid \lambda \in \mathbb{R}_{+}^{(T)}\right\} .
$$

As a consequence of Proposition 5.1, we have e'co $K \subset$ epi $\left(f+\delta_{A}\right)^{c}$. Now, if we prove that, for all $x \in X$,

we will obtain that

$$
\left(f+\delta_{A}\right)(x) \leq h^{c^{\prime}}(x)
$$

$$
h^{c^{\prime} c}\left(x^{*}, y^{*}, \alpha\right) \leq\left(f+\delta_{A}\right)^{c}\left(x^{*}, y^{*}, \alpha\right)
$$

for all $\left(x^{*}, y^{*}, \alpha\right) \in W$, and hence epi $\left(f+\delta_{A}\right)^{c} \subset$ epi $h^{c^{\prime} c}=\mathrm{e}^{\prime} \operatorname{co} K$.

Then, take any point $x \in X$. Recalling that, in our context, $f$ and $g_{t}$, for all $t \in T$ are e-convex, hence for all $\lambda \in \mathbb{R}_{+}^{(T)}, f+\lambda g$ is e-convex, we have

$$
\begin{aligned}
h^{c^{\prime}}(x) & =\sup _{\left(x^{*}, y^{*}, \alpha\right) \in W}\left\{c\left(x,\left(x^{*}, y^{*}, \alpha\right)\right)-\inf _{\lambda \in \mathbb{R}_{+}^{(T)}}(f+\lambda g)^{c}\left(x^{*}, y^{*}, \alpha\right)\right\} \\
& =\sup _{\substack{\left(x^{*}, y^{*}, \alpha\right) \in W \\
\lambda \in \mathbb{R}_{+}^{(T)}}}\left\{c\left(x,\left(x^{*}, y^{*}, \alpha\right)\right)-(f+\lambda g)^{c}\left(x^{*}, y^{*}, \alpha\right)\right\} \\
& =\sup _{\lambda \in \mathbb{R}_{+}^{(T)}}(f+\lambda g)^{c^{\prime} c}(x)=\sup _{\lambda \in \mathbb{R}_{+}^{(T)}}(f+\lambda g)(x) .
\end{aligned}
$$

Now, if $x \in A,\left(f+\delta_{A}\right)(x)=f(x)$ and

$$
h^{c^{\prime}}(x)=\sup _{\lambda \in \mathbb{R}_{+}^{(T)}}(f+\lambda g)(x) \geq f(x) .
$$

In other case, $\left(f+\delta_{A}\right)(x)=+\infty$. Since $x \notin A$, there exists $\widehat{t} \in T$ verifying $g_{\widehat{t}}(x)>0$. Taking

$$
\mathbb{R}_{+}^{(T)} \supset\left(\lambda_{r}\right):=\left\{\begin{array}{cc}
r, & \text { if } t=\widehat{t} \\
0, & \text { otherwise }
\end{array}\right.
$$

we have $\left(f+\lambda_{r} g\right)(x)=f(x)+r g_{\hat{t}}(x)$ which goes to infinite when $r \rightarrow \infty$, and hence $\sup _{\lambda \in \mathbb{R}_{+}^{(T)}}(f+\lambda g)(x)=+\infty$. we obtain that, in any case, $\left(f+\delta_{A}\right)(x) \leq h^{c^{\prime}}(x)$. 
Let us denote

(C5) $\bigcup_{\lambda \in \mathbb{R}_{+}^{(T)}} \operatorname{epi}(f+\lambda g)^{c}$ is an $\mathrm{e}^{\prime}$-convex set.

Corollary 5.1 Condition (C5) implies condition (C2), hence it is also a regularity condition for strong duality and a stable strong sufficient condition.

We finish showing that, unfortunately, (C5) does not characterize stable strong Lagrange duality, since the following example allows us to see that $\left(\mathrm{C}_{L}\right)$ does not imply $(\mathrm{C} 5)$. Hence, our objective of characterizing such duality remains opened.

Example 5.1 Let us take $X=\mathbb{R}, f=\delta_{[0,+\infty}$ and $\sigma=\left\{t x+\delta_{]-\infty, t]}(x) \leq 0, t \in T\right\}$, being $T=[0,+\infty[$. We have $A=]-\infty, 0]$. In Example 5.2 from [7], it is showed that $\left(\mathrm{C}_{L}\right)$ holds, and, moreover,

We are going to see that

$$
\text { epi } f+\operatorname{epi} \delta_{A}^{c}=\mathbb{R} \times \mathbb{R} \times \mathbb{R}_{++} \times \mathbb{R}_{+} \text {. }
$$

$$
\bigcup_{\lambda \in \mathbb{R}_{+}^{(T)}} \operatorname{epi}(f+\lambda g)^{c} \varsubsetneqq \mathrm{e}^{\prime} \text { co } \bigcup_{\lambda \in \mathbb{R}_{+}^{(T)}} \operatorname{epi}(f+\lambda g)^{c},
$$

being, in this case, e'co $\bigcup_{\lambda \in \mathbb{R}_{+}^{(T)}} \operatorname{epi}(f+\lambda g)^{c}=\operatorname{epi}\left(f+\delta_{A}\right)^{c}=\operatorname{epi} f^{c}+\operatorname{epi} \delta_{A}^{c}$.

Let us take any $\lambda \in \mathbb{R}_{+}^{(T)}$. Then $\left(y^{*}, z^{*}, \alpha, \beta\right) \in \operatorname{epi}(f+\lambda g)^{c}$ if and only if, for all $x \in X$,

$$
c\left(x,\left(y^{*}, z^{*}, \alpha\right)\right)-f(x)-\lambda g(x) \leq \beta .
$$

It is equivalent to the fulfilment of,

$$
\left\langle x, y^{*}\right\rangle-\sum_{t \in \operatorname{supp} \lambda} \lambda_{t}\left(t x+\delta_{]-\infty, t]}(x)\right) \leq \beta \text { and }\left\langle x, z^{*}\right\rangle<\alpha,
$$

for all $x \geq 0$. It implies, in particular, that $z^{*} \leq 0$, and it happens for any $\lambda \in \mathbb{R}$. Then

$$
\bigcup_{\lambda \in \mathbb{R}_{+}^{(T)}} \operatorname{epi}(f+\lambda g)^{c} \varsubsetneqq \mathbb{R} \times \mathbb{R} \times \mathbb{R}_{++} \times \mathbb{R}_{+} .
$$

\section{References}

[1] Bot,, R.I., Grad, S., Wanka, G. (2008) New regularity conditions for strong and total FenchelLagrange duality in infinite dimensional spaces.Nonlinear Analysis 69: 323-336.

[2] Burachik, R.S., Jeyakumar, V., Wu, Z.-Y. (2006) Necessary and sufficient conditions for stable conjugate duality. Nonlinear Analysis, 64:1998-2006. 
[3] Daniilidis, A., Martínez-Legaz, J.E. (2002) Characterizations of evenly convex sets and evenly quasiconvex functions. J. Mathematical Analysis and Applications 273: 58-66.

[4] Fenchel, W. (1952) A remark on convex sets and polarity. Comm. Sèm. Math. Univ. Lund (Medd. Lunds Algebra Univ. Math. Sem.), Tome Supplémentaire, 82-89.

[5] Fajardo, M.D. (2015) Regularity conditions for strong duality in evenly convex optimization problems. An application to Fenchel duality. Journal of Convex Analysis, 22 (3), available in electronic form.

[6] Fajardo, M.D., Vicente-Pérez, J., Rodríguez, M.M.L.(2012) Infimal convolution, c-subdifferentiability and Fenchel duality in evenly convex optimization. TOP 20 (2): 375-396.

[7] Fajardo, M.D., Rodríguez, M.M.L.Vidal, J., (2015) Strong Lagrange duality for evenly convex optimization problems. Submitted.

[8] Goberna, M.A., Jeyakumar, V., López, M.A. (2008) Necessary and sufficient constraint qualifications for solvability of systems of infinite convex inequalities. Nonlinear Analysis: Theory, Methods \& Applications. Vol. 68, No. 5, pp. 1184-1194.

[9] Goberna, M.A., Jornet, V., Rodríguez, M.M.L. (2003) On linear systems containing strict inequalities. Linear Algebra and its Applications 360: 151-171.

[10]Goberna, M.A., Rodríguez, M.M.L. (2006) Analyzing linear systems containing strict inequalities via evenly convex hulls. European Journal of Operational Research 169: 10791095.

[11]Li, C., Fang, D., López, G., López, M.A. (2009) Stable and total Fenchel duality for convex optimization problems in locally convex spaces. SIAM J. Optim., 20 (2): 1032-1051

[12]Klee, V., Maluta, E., Zanco, C. (2007) Basic properties of evenly convex sets. Journal of Convex Analysis 14 (1): 137-148.

[13]Martínez-Legaz, J.E. (1988) Quasiconvex duality theory by generalized conjugation methods. Optimization 19: 603-652.

[14]Martínez-Legaz, J.E. (2005) Generalized convex duality and its economic applications. In: Hadjisavvas, N., Komlósi, S., Schaible, S. (eds) Handbook of Generalized Convexity and Generalized Monotonicity, Springer, New York, pp. 237-292.

[15]Martínez-Legaz, J.E., Vicente-Pérez, J. (2011) The e-support function of an e-convex set and conjugacy for e-convex functions. J. Math. Anal. Appl. 376: 602-612. 
[16]J.J. Moreau (1970) Inf-convolution, sous-additivité, convexité des fonctions numériques. J. Math. Pures et Appl. 49: 109-154.

[17]Rodríguez, M.M.L., Vicente-Pérez, J. (2011) On evenly convex functions. Journal of Convex Analysis 18: 721-736.

[18]Zălinescu, C. (2002) Convex analysis in general vector spaces. World Scientific, New Jersey. 